\title{
Antipsychotic Adherence and Rehospitalization in Schizophrenia Patients Receiving Oral Versus Long-Acting Injectable Antipsychotics Following Hospital Discharge
}

\author{
Steven C. Marcus, PhD; Jacqueline Zummo, MPH, MBA; Amy R. Pettit, PhD; \\ Jeffrey Stoddard, MD; and Jalpa A. Doshi, PhD
}

\begin{abstract}
BACKGROUND: Antipsychotic medications are a central component of effective treatment for schizophrenia, but nonadherence is a significant problem for the majority of patients. Long-acting injectable (LAI) antipsychotic medications are a recommended treatment option for nonadherent patients, but evidence regarding their potential advantages has been mixed. Observational data on newer, second-generation LAI antipsychotic medications have been limited given their more recent regulatory approval and availability.
\end{abstract}

OBJECTIVE: To examine antipsychotic medication nonadherence, discontinuation, and rehospitalization outcomes in Medicaid patients receiving oral versus LAI antipsychotic medications in the 6 months after a schizophrenia-related hospitalization.

METHODS: The 2010-2013 Truven Health Analytics MarketScan Medicaid research claims database was used to identify adult patients with a recent history of nonadherence (prior 6 months) who received an oral or LAI antipsychotic medication within 30 days after an index schizophrenia-related hospitalization. Primary outcome measures were nonadherence (proportion of days covered $<0.80$ ), discontinuation (continuous medication gap $\geq 60$ days), and schizophrenia-related rehospitalization, all in the 6 months after discharge. Descriptive analyses compared users of oral versus LAI antipsychotic medication on sociodemographic, clinical, and treatment characteristics. Logistic regressions were used to examine associations between use of oral versus LAI antipsychotics and each study outcome while controlling for observed differences in sample characteristics. All outcomes were compared at 3 levels of analysis: overall LAI class, LAI antipsychotic generation (first-generation [FGA] or second-generation [SGA] antipsychotics), and individual LAI agent (fluphenazine decanoate, haloperidol decanoate, risperidone LAI, and paliperidone palmitate).

RESULTS: Of the final sample, $91 \%(n=3,428)$ received oral antipsychotics, and $9.0 \%(n=340)$ received LAl antipsychotics after discharge. Slightly over half $(n=183,53.8 \%)$ of LAl users used an SGA LAI. A smaller percentage of patients receiving LAls were nonadherent ( $51.8 \%$ vs. $67.7 \%, P<0.001)$; had a 60 -day continuous gap in medication $(23.8 \%$ vs. $39.4 \%, P<0.001)$; and were rehospitalized for schizophrenia ( $19.1 \%$ vs. $25.3 \%, P=0.01)$ compared with patients receiving oral medications. The size of these differences was magnified when comparing SGA LAI users with users of oral antipsychotics for nonadherence. After controlling for all differences in measured covariates, LAI initiators had lower odds of being nonadherent (adjusted odds ratio $[\mathrm{AOR}]=0.35,95 \% \mathrm{Cl}=0.27-0.46, P<0.001$ ) and of having continuous 60 -day gaps $(\mathrm{AOR}=0.45,95 \% \mathrm{Cl}=0.34-0.60, P<0.001)$ when compared with patients receiving oral medications. Both FGA and SGA LAI users had lower odds of nonadherence compared with patients receiving oral antipsychotics. Similarly, FGA LAI users (AOR $=0.58,95 \% \mathrm{Cl}=0.40-0.85$, $P=0.005)$ and SGA LAI initiators $(\mathrm{AOR}=0.34,95 \% \mathrm{Cl}=0.23-0.51, P<0.001)$ had lower odds of a 60 -day continuous gap compared with patients receiving oral antipsychotics. Compared with those receiving oral antipsychotics, LAl initiators also had lower odds of rehospitalization (AOR $=0.73$, $95 \% \mathrm{Cl}=0.54-0.99, P=0.041)$; however, when examined separately, only patients receiving SGA LAls (AOR $=0.59,95 \% \mathrm{Cl}=0.38-0.90, P=0.015)$ and not FGA LAls ( $A O R=0.90,95 \% \mathrm{Cl}=0.60-1.34, P=0.599)$ had a statistically significant reduction in odds of rehospitalization. Among individual LAls, odds of rehospitalization only among initiators of paliperidone palmitate were statistically different from those among users of oral antipsychotics (AOR $=0.53,95 \% \mathrm{Cl}=0.30-0.94, P=0.031$ ). While odds of rehospitalization were $33 \%$ lower among patients receiving risperidone LAI compared with those receiving oral antipsychotics, the estimate did not reach statistical significance $(\mathrm{AOR}=0.67,95 \% \mathrm{Cl}=0.37-1.22, P=0.194)$.

CONCLUSIONS: This claims-based analysis of posthospitalization adherence and rehospitalization outcomes in Medicaid patients with schizophrenia adds to the growing real-world evidence base of the benefits of LAI antipsychotic medications in routine clinical practice, particularly with regard to second-generation LAls. As new SGA formulations become available for long-acting use, real-world studies with larger sample sizes will be needed to further delineate their potential advantages in terms of clinical outcomes and costs.

\section{J Manag Care Spec Pharm. 2015;21(9):754-68}

Copyright $\odot 2015$, Academy of Managed Care Pharmacy. All rights reserved.

\section{What is already known about this subject}

Schizophrenia is typically a chronic, disabling psychiatric disorder with tremendous human and financial costs. Antipsychotic medications are a central component of effective treatment, but nonadherence is a significant problem for the majority of patients. Once-monthly or bi-weekly long-acting injectable (LAI) medications offer reduced adherence demands compared with daily oral medications, and multiple observational, "real-world" studies have demonstrated that patients on LAI therapy have a reduced risk of relapse and hospitalization. However, observational data on newer, second-generation LAI antipsychotic medications is limited given their more recent regulatory approval and availability. 


\section{Antipsychotic Adherence and Rehospitalization in Schizophrenia Patients Receiving Oral Versus Long-Acting Injectable Antipsychotics Following Hospital Discharge}

\section{What this study adds}

This claims-based study builds on prior analyses of utilization outcomes associated with use of oral versus LAI antipsychotics by including second-generation LAI medications that have more recently entered the market and by examining a multistate Medicaid population with a recent history of nonadherence.

Following discharge from a hospitalization for schizophrenia, patients who received LAIs were more likely to be adherent and were less likely to have a 60-day continuous gap in available medication, compared with patients receiving oral antipsychotic medications; second-generation LAI therapy showed more pronounced advantages relative to oral medications.

Paliperidone palmitate was the only LAI agent associated with a statistically significant reduction in risk for rehospitalization relative to patients receiving oral antipsychotics, but the ability to detect a similar statistically significant advantage for risperidone LAI may have been limited by its smaller sample size.

S chizophrenia is a serious and persistent psychiatric disorder associated with substantial clinical, personal, and economic burdens. ${ }^{1-3}$ Consistent use of antipsychotic medications is central to effective management of schizophrenia symptoms, ${ }^{4-6}$ yet numerous U.S. studies have documented poor antipsychotic medication adherence in patients with schizophrenia. ${ }^{7,8}$ Low medication persistence is also a widespread issue. The Clinical Antipsychotic Trials of Intervention Effectiveness (CATIE) study, a large pragmatic trial conducted in the United States, found that $74 \%$ of patients receiving oral antipsychotics discontinued the study medication within 18 months. ${ }^{9}$ Moreover, a substantial body of data has linked medication nonadherence to relapse and the "revolving door" phenomenon of frequent psychiatric hospitalizations..$^{10-12}$ These repeated hospitalizations are disruptive to individuals and families and costly for the health care system.

Long-acting injectable (LAI) antipsychotic medications, which are typically administered on a biweekly or monthly basis as opposed to the daily dosing required for oral medications, were developed to address nonadherence in patients with chronic psychosis. ${ }^{13,14}$ They ensure medication delivery for a specified period of time, thereby eliminating questions about whether medication has been taken as prescribed. LAIs also eliminate the adherence demands of daily dosing, which can pose particular challenges due to the impaired thinking, memory difficulties, and lack of insight common in schizophrenia. ${ }^{4,15,16}$ LAI therapy also makes early detection of nonadherence simple (i.e., synonymous with a missed visit for receiving the injection), whereas nonadherence to oral medications often goes undetected until a major problem develops. ${ }^{17}$ Clinical practice guidelines highlight LAI therapy as an appropriate option for patients with a significant history of nonadherence. ${ }^{5,6,18}$

Although LAI formulations have been shown to reduce relapse risk in clinical trials when compared with placebo, randomized controlled trials (RCTs) comparing oral and LAI antipsychotics have often failed to show advantages of LAIs over oral antipsychotics in terms of relapse and/or hospitalization risk. ${ }^{19}$ However, the results of these RCTs must be interpreted in the context of several limitations. ${ }^{20}$ RCTs involve more homogeneous groups of patients, often with less severe symptoms or fewer comorbidities, and exclude many patients treated for schizophrenia in routine practice. ${ }^{21}$ In particular, patients with adherence problems are likely to be excluded from or reluctant to participate in clinical trials. The frequent (often biweekly) monitoring intrinsic to RCTs also may encourage adherence or detect early nonadherence more effectively than is the case in usual care. Since these effects of trial participation on adherence would introduce bias favoring the oral medication group, RCTs may systematically underestimate advantages of LAIs that might be seen with actual use in clinical practice. ${ }^{22,23}$ Furthermore, most clinical trials, including the recent pragmatic randomized trial PROACTIVE, ${ }^{23}$ included the second-generation antipsychotic (SGA) LAI risperidone (Risperdal Consta) ${ }^{24}$ but did not incorporate more recently approved SGA LAIs that typically require monthly as opposed to biweekly administrations. ${ }^{19}$ Hence, studies are also needed to examine whether the more recently available SGA LAIs confer advantages over oral antipsychotics in terms of improved adherence and lower risk of schizophrenia-related hospitalizations among nonadherent patients found in real-world settings.

Most previous real-world studies using a pre-post or mirror image study design have found a beneficial effect of LAIs over oral antipsychotics in terms of reductions in rehospitalizations, but the lack of a control group is a serious methodological limitation of this evidence base. ${ }^{14,25}$ The few observational studies that have used administrative databases and included a comparison group have also demonstrated advantages of LAIs over oral antipsychotics, but they are limited in terms of the generalizability of their study populations, the specific SGA agents included in the LAI group, and/or their level of detail in separating out treatment effects by type of LAI (i.e., firstgeneration antipsychotics [FGA] vs. SGA) and by individual LAI agents. ${ }^{26-30}$

Our study aimed to examine antipsychotic adherence and rehospitalization in patients with schizophrenia who had a recent history of nonadherence to oral antipsychotics and received oral versus LAI antipsychotics after hospital discharge. We sought to address limitations in the existing evidence base in several key ways. First, we used more recent data so as to allow inclusion of SGA LAI options that have been approved since the introduction of risperidone LAI in 2003. In particular, paliperidone palmitate (Invega Sustenna) has a monthly dosing schedule after the initial loading doses and has been examined in few studies. ${ }^{27,30-32}$ 


\section{Antipsychotic Adherence and Rehospitalization in Schizophrenia Patients Receiving Oral Versus Long-Acting Injectable Antipsychotics Following Hospital Discharge}

Second, we reported detailed data on outcomes for patients receiving oral medications compared with those receiving LAI therapy at 3 levels of analysis: the overall LAI class level, the LAI antipsychotic generation (FGA or SGA) level, and the individual LAI agent level.

Third, we sought to extend this investigation to a multistate Medicaid population, given that these individuals have not been included in most prior studies comparing oral and LAI antipsychotics. Although schizophrenia is estimated to affect approximately $1 \%$ of the general population, an estimated $1.6 \%$ of Medicaid patients carry the diagnosis. ${ }^{33}$ Since the onset of the disorder occurs most frequently in young adulthood and often leads to profound interference with employment, it is not surprising that almost a third of schizophrenia patients in the United States qualify for the low-income Medicaid program. ${ }^{2,9,33}$ Given these contextual considerations, investigation of whether LAIs confer advantages over oral antipsychotics in this vulnerable population is warranted.

Finally, we examined a group of patients who represent a target population for LAI use, namely individuals with a schizophrenia-related hospitalization following a recent history of nonadherence to oral antipsychotic medication. By requiring all patients to meet these criteria, we sought to level the playing field at baseline - to the extent possible-between oral and LAI users in this observational administrative claims study.

\section{Methods}

\section{Data Source}

This study utilized administrative claims data drawn from the Truven Health Analytics MarketScan Medicaid research claims database between January 1, 2010, and July 31, 2013. The database includes information from multiple state Medicaid programs and includes demographic and clinical information, inpatient and outpatient utilization data, and outpatient prescription data for Medicaid enrollees. In constructing the database, encounter records are rigorously tested by means of overall plan-by-plan utilization rates to ensure that plans appearing to submit incomplete data are excluded. All personally identifiable patient, provider, and facility data were replaced with fully de-identified markers, in conformance with the Health Insurance Portability and Accountability Act of 1996, prior to delivery for research use. This included removal of any state identifiers and all other geographic identifiers to prevent identification of the individual state Medicaid programs contributing to the database. No data were collected directly from human subjects; thus, institutional review board approval was not required.

\section{Study Design and Sample Selection}

This observational study employed a retrospective cohort design using administrative claims data. Primary inclusion criteria captured nondual eligible Medicaid adults who (a) were discharged to the community following an index hospitalization, defined as the first hospitalization with a primary diagnosis of schizophrenia (International Classification of Diseases, Ninth Revision, Clinical Modification [ICD-9-CM] code 295.xx ${ }^{34}$ between July 1, 2010, and December 31, 2012; (b) were aged 18 years or older on the index hospitalization date; (c) had continuous Medicaid coverage including mental health and prescription benefits and were not in a behavioral health carve out for 6 months before and after the index hospitalization, to ensure complete claims information; (d) had poor adherence to oral antipsychotic medication in the 6 months prior to index hospitalization, defined as proportion of days covered (PDC) less than 0.80 (see "Key Outcome Measures" section); (e) had no use of clozapine prior to hospitalization, to exclude treatment-resistant patients; (f) had no use of an LAI prior to hospitalization, to identify new users of LAIs after hospital discharge; (g) received an oral or LAI antipsychotic medication within 30 days of hospital discharge; and (h) were not rehospitalized prior to receiving the oral or LAI antipsychotic medication during the 30 days after discharge, to exclude cases where the outcome preceded the exposure. By defining our sample based on an index hospitalization, we were able to focus on a key prescribing decision point and to measure adherencerelated outcomes during a distinct phase of treatment after hospital discharge.

We also sought to reduce the likelihood of selection bias between the oral and LAI groups by requiring all patients to have evidence of nonadherence to oral antipsychotic medication during the pre-index period, since adherent patients may be less likely to be considered candidates for LAIs. Figure 1 provides additional details of the sample selection process.

The LAI group was further divided by FGA and SGA LAIs, as well as into subgroups according to specific LAI agent (fluphenazine decanoate, haloperidol decanoate, risperidone LAI, and paliperidone palmitate). Although patients on all SGA LAIs approved during the study period were eligible for inclusion, no patients on olanzapine pamoate (Zyprexa Relprevv) met all eligibility criteria. ${ }^{35}$ Aripiprazole LAI (Abilify Maintena), approved by the U.S. Food and Drug Administration in 2013, was not yet available during our study sample selection period. $^{36}$

The control group for all sets of primary analyses consisted of all patients receiving an oral antipsychotic. In sensitivity analyses, patients receiving FGA orals were used as a control group for comparisons with FGA LAIs (overall and by individual FGA LAI agent), and patients receiving SGA orals were used as a control group for comparisons with SGA LAIs (overall and by individual SGA LAI agent).

\section{Key Outcome Measures}

Antipsychotic Adherence. Medication-taking behavior was captured using PDC methodology, which is calculated by 
Antipsychotic Adherence and Rehospitalization in Schizophrenia Patients Receiving

Oral Versus Long-Acting Injectable Antipsychotics Following Hospital Discharge

\section{FIGURE 1 Sample Selection Flowchart}

Medicaid patients in claims database with eligible index hospitalization (primary diagnosis of schizophrenia, July 1, 2010-December 31, 2012)

$(\mathrm{N}=24,599)$

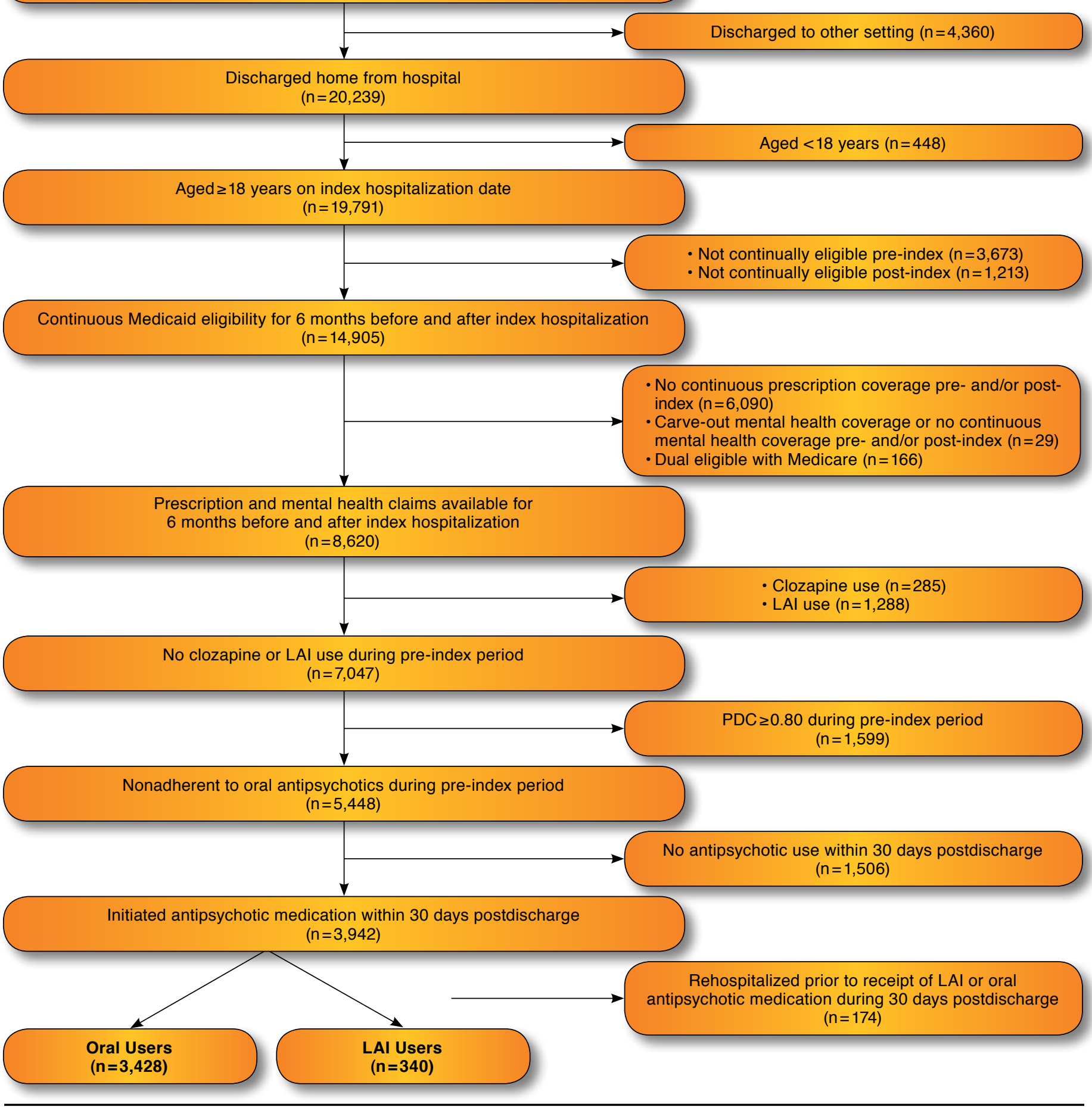

LAI=long-acting injectable; $P D C=$ proportion of days covered 


\section{Antipsychotic Adherence and Rehospitalization in Schizophrenia Patients Receiving Oral Versus Long-Acting Injectable Antipsychotics Following Hospital Discharge}

TABLE 1 Baseline Characteristics of Patients with Schizophrenia Receiving Oral Versus LAI Medications, Overall and by Antipsychotic Class ${ }^{\mathrm{a}}$

\begin{tabular}{|c|c|c|c|c|c|c|c|c|c|c|c|}
\hline \multirow[b]{2}{*}{ Variable } & \multicolumn{2}{|c|}{ Orals $(\mathrm{n}=3,428)$} & \multicolumn{3}{|c|}{ LAIs $(n=340)$} & \multicolumn{3}{|c|}{ FGA LAIs $(\mathrm{n}=157)$} & \multicolumn{3}{|c|}{ SGA LAIs $(n=183)$} \\
\hline & $\%$ & (n) & $\%$ & (n) & $P$ Value & $\%$ & (n) & $P$ Value & $\%$ & (n) & $P$ Value \\
\hline \multicolumn{12}{|l|}{ Age, years } \\
\hline Mean [SD] & 38.0 & [12.9] & 37.5 & {$[13.8]$} & 0.554 & 38.7 & [12.9] & 0.482 & 36.5 & {$[14.5]$} & 0.172 \\
\hline Median [IQR] & 37.0 & {$[23.0]$} & 35.0 & {$[23.0]$} & 0.346 & 39.0 & {$[21.0]$} & 0.504 & 32.0 & {$[23.0]$} & 0.054 \\
\hline Gender & & & & & $<0.001$ & & & 0.025 & & & $<0.001$ \\
\hline Female & 51.2 & $(1,755)$ & 39.1 & (133) & & 42.0 & (66) & & 36.6 & (67) & \\
\hline Male & 48.8 & $(1,673)$ & 60.9 & (207) & & 58.0 & (91) & & 63.4 & (116) & \\
\hline Race & & & & & 0.008 & & & 0.001 & & & 0.012 \\
\hline African American & 48.2 & $(1,652)$ & 55.0 & (187) & & 65.0 & $(102)$ & & 46.4 & $(85)$ & \\
\hline White & 42.6 & $(1,460)$ & 34.7 & (118) & & 26.1 & (41) & & 42.1 & (77) & \\
\hline Hispanic & 1.1 & $(37)$ & 2.4 & (8) & & 0.6 & (1) & & 3.8 & (7) & \\
\hline Other & 8.1 & $(279)$ & 7.9 & (27) & & 8.3 & (13) & & 7.7 & (14) & \\
\hline Type of Medicaid plan & & & & & 0.552 & & & 0.021 & & & 0.003 \\
\hline Fee-for-service & 58.9 & $(2,019)$ & 60.6 & $(206)$ & & 49.7 & $(78)$ & & 69.9 & $(128)$ & \\
\hline Capitated & 41.1 & $(1,409)$ & 39.4 & (134) & & 50.3 & (79) & & 30.1 & $(55)$ & \\
\hline \multicolumn{12}{|l|}{ Number of Elixhauser comorbidities, pre-index } \\
\hline Mean [SD] & 2.8 & [2.2] & 2.5 & {$[2.1]$} & 0.004 & 2.5 & [2.0] & 0.027 & 2.5 & {$[2.1]$} & 0.046 \\
\hline Median [IQR] & 2.0 & [3.0] & 2.0 & {$[3.0]$} & 0.002 & 2.0 & {$[2.0]$} & 0.017 & 2.0 & {$[3.0]$} & 0.029 \\
\hline \multicolumn{12}{|l|}{ Specific comorbidities, pre-index } \\
\hline Diabetes/hypertension/circulatory disorders & 39.0 & $(1,337)$ & 35.3 & $(120)$ & 0.181 & 37.6 & (59) & 0.721 & 33.3 & (61) & 0.125 \\
\hline Injury & 31.2 & $(1,069)$ & 25.6 & $(87)$ & 0.033 & 20.4 & $(32)$ & 0.004 & 30.1 & (55) & 0.748 \\
\hline Bipolar disorder & 34.1 & $(1,170)$ & 26.5 & (90) & 0.004 & 25.5 & (40) & 0.025 & 27.3 & (50) & 0.058 \\
\hline Depression & 39.2 & $(1,344)$ & 25.9 & (88) & $<0.001$ & 27.4 & (43) & 0.003 & 24.6 & (45) & $<0.001$ \\
\hline Anxiety & 34.4 & $(1,178)$ & 23.8 & $(81)$ & $<0.001$ & 21.0 & $(33)$ & $<0.001$ & 26.2 & $(48)$ & 0.024 \\
\hline Substance abuse & 44.0 & $(1,507)$ & 45.3 & (154) & 0.637 & 45.2 & $(71)$ & 0.756 & 45.4 & (83) & 0.711 \\
\hline Suicidal behavior and/or ideation & 17.3 & (593) & 10.6 & $(36)$ & 0.002 & 10.8 & $(17)$ & 0.035 & 10.4 & (19) & 0.015 \\
\hline \multicolumn{12}{|l|}{ Oral antipsychotic adherence, pre-index } \\
\hline Mean PDC [SD] & 0.28 & {$[0.28]$} & 0.23 & {$[0.26]$} & 0.001 & 0.21 & {$[0.25]$} & 0.001 & 0.25 & {$[0.27]$} & 0.099 \\
\hline Any 60-day gap & 69.8 & $(2,394)$ & 77.6 & (264) & 0.490 & 79.6 & $(125)$ & 0.727 & 76.0 & (139) & 0.463 \\
\hline \multicolumn{12}{|l|}{ Oral antipsychotic agents used, pre-index } \\
\hline Fluphenazine oral & 1.2 & $(40)$ & 2.4 & (8) & 0.063 & 4.5 & $(7)$ & $<0.001$ & 0.5 & (1) & 0.544 \\
\hline Haloperidol oral & 7.9 & $(270)$ & 10.3 & (35) & 0.119 & 14.0 & $(22)$ & 0.006 & 7.1 & (13) & 0.705 \\
\hline Other FGA oral & 5.2 & (179) & 5.6 & (19) & 0.773 & 5.1 & $(8)$ & 0.945 & 6.0 & (11) & 0.641 \\
\hline Olanzapine oral & 8.7 & $(297)$ & 9.4 & (32) & 0.641 & 8.9 & (14) & 0.912 & 9.8 & (18) & 0.584 \\
\hline Paliperidone oral & 4.2 & (144) & 3.8 & (13) & 0.740 & 1.3 & (2) & 0.091 & 6.0 & (11) & 0.239 \\
\hline Quetiapine oral & 20.0 & (684) & 15.6 & (53) & 0.053 & 16.6 & (26) & 0.297 & 14.8 & (27) & 0.085 \\
\hline Ziprasidone oral & 9.0 & (310) & 6.2 & (21) & 0.075 & 7.0 & (11) & 0.382 & 5.5 & (10) & 0.097 \\
\hline Aripiprazole oral & 9.8 & (336) & 6.8 & (23) & 0.069 & 5.7 & (9) & 0.091 & 7.7 & (14) & 0.338 \\
\hline Risperidone oral & 20.8 & $(713)$ & 21.8 & (74) & 0.676 & 15.9 & $(25)$ & 0.140 & 26.8 & (49) & 0.054 \\
\hline Other SGA oral & 3.1 & $(107)$ & 3.2 & (11) & 0.908 & 2.5 & (4) & 0.820 & 3.8 & (7) & 0.596 \\
\hline \multicolumn{12}{|l|}{ Other CNS drug use, pre-index } \\
\hline Other MH drug use, pre-index ${ }^{b}$ & 59.3 & $(2,032)$ & 48.2 & (164) & $<0.001$ & 45.9 & $(72)$ & $<0.001$ & 50.3 & (92) & 0.016 \\
\hline Anticholinergic use, pre-index & 21.3 & $(731)$ & 25.3 & $(86)$ & 0.090 & 30.6 & $(48)$ & 0.006 & 20.8 & (38) & 0.857 \\
\hline Any MH hospitalization, pre-index & 19.8 & $(680)$ & 16.5 & (56) & 0.135 & 19.1 & (30) & 0.827 & 14.2 & (26) & 0.061 \\
\hline Any non-MH hospitalization, pre-index & 12.5 & $(429)$ & 9.7 & (33) & 0.132 & 8.3 & (13) & 0.134 & 10.9 & (20) & 0.526 \\
\hline Year of index hospitalization & & & & & $<0.001$ & & & $<0.001$ & & & $<0.001$ \\
\hline 2010 & 39.5 & $(1,353)$ & 39.4 & $(134)$ & & 44.6 & $(70)$ & & 35.0 & (64) & \\
\hline 2011 & 30.8 & $(1,055)$ & 28.8 & (98) & & 28.0 & (44) & & 29.5 & (54) & \\
\hline 2012 & 29.8 & $(1,020)$ & 31.8 & $(108)$ & & 27.4 & $(43)$ & & 35.5 & $(65)$ & \\
\hline \multicolumn{12}{|l|}{ Index hospitalization length of stay, days } \\
\hline Mean [SD] & 8.9 & [9.2] & 12.8 & {$[12.0]$} & $<0.001$ & 12.1 & {$[8.5]$} & $<0.001$ & 13.4 & [14.4] & $<0.001$ \\
\hline Median [IQR] & 7.0 & [5.0] & 10.0 & {$[8.0]$} & $<0.001$ & 9.0 & [8.0] & $<0.001$ & 10.0 & {$[8.0]$} & $<0.001$ \\
\hline \multicolumn{12}{|c|}{$\begin{array}{l}\text { Includes patients receiving an antipsychotic medication within } 30 \text { days of discharge from index schizophrenia-related hospitalization. Baseline refers to the index hospital- } \\
\text { ization date and the prior } 6 \text {-month pre-index period. P values were calculated with chi-square tests for categorical variables, } t \text {-tests or Fisher's exact tests (for cell sizes less } \\
\text { than 5) for continuous variables, and Wilcoxon tests for medians. } \\
\text { bIncludes antidepressants, anxiolytics, mood stabilizers, sedatives, and hypnotics. } \\
C N S=\text { central nervous system; FGA = first-generation antipsychotic; IQR=interquartile range; LAI=long-acting injectable; MH=mental health; PDC=proportion of days } \\
\text { covered; SD = standard deviation; SGA=second-generation antipsychotic. }\end{array}$} \\
\hline
\end{tabular}




\section{Antipsychotic Adherence and Rehospitalization in Schizophrenia Patients Receiving Oral Versus Long-Acting Injectable Antipsychotics Following Hospital Discharge}

dividing the number of days with medication available by the number of days in a given time interval. ${ }^{37}$ For all oral antipsychotics, the days' supply as reported on the prescription claim was used to calculate the PDC. Given that the days' supply field is unavailable or of questionable accuracy for LAI antipsychotics in medical or prescription claims, respectively, the days' supply on each claim was set to the minimum of time between injections and the time frame as per the labeled dosing schedule for the given injectable. ${ }^{38}$ We calculated PDC for each patient in the 6-month period after hospital discharge; patients with a PDC less than 0.80 were deemed nonadherent.

Antipsychotic Discontinuation. Discontinuation was defined as a dichotomous measure, reflecting the presence or absence of a continuous gap of 60 days or more in the available days' supply of antipsychotic medication during the 6-month postindex period. Patients with 1 or more continuous 60-day gaps were considered as having discontinued the medication. ${ }^{39,40}$ In sensitivity analyses, we varied the length of the continuous gap used to define discontinuation to 30 and 90 days.

Rehospitalization. The primary outcome of interest was any schizophrenia-related rehospitalization, defined as any hospitalization with a primary diagnosis of schizophrenia (ICD9-CM code 295.xx), during the 6-month post-index period. In sensitivity analyses, we also examined any mental healthrelated rehospitalizations (ICD-9-CM codes 290.xx-319.xx) and all-cause rehospitalizations.

\section{Control Variables}

Variables that are potentially related to illness severity and adherence behavior were also examined. These included sociodemographics (age, sex, race, and type of Medicaid plan); comorbidities identified during the pre-index period (number of Elixhauser comorbidities, ${ }^{41}$ diabetes mellitus/hypertension/ circulatory disorders, injury, bipolar disorder, depression, anxiety, substance abuse, and suicidal behavior or ideation); use of other mental health drugs (antidepressants, anxiolytics, mood stabilizers, sedatives, and hypnotics) and anticholinergic agents during the pre-index period; specific oral antipsychotic agent used during the pre-index period; adherence as measured by PDC and any 60-day continuous gap in use; any mental health or other hospitalization in the pre-index period; and characteristics of the index hospitalization (year and length of stay). All regressions also controlled for the time (days) between index hospital discharge date and the date when the first antipsychotic (oral or LAI) was received during the first 30 days after discharge from the index hospitalization.

\section{Statistical Analysis}

We generated descriptive statistics to characterize each group of interest (users of orals, any LAI, FGA or SGA LAIs, and individual LAI agents postdischarge). Group comparisons on baseline sample characteristics were performed using chisquare tests for categorical variables and t-tests or Fisher's exact tests for continuous variables. Wilcoxon tests were used to examine differences in medians. Logistic regressions were used to examine the association between the oral and LAI antipsychotics and each of the study outcomes (i.e., adherence, discontinuation, and rehospitalization) while controlling for observed differences across groups for all covariates mentioned above. Three sets of regression models were estimated for each outcome wherein patients receiving oral antipsychotics were compared with users of (1) any LAI, (2) FGA and SGA LAIs, and (3) individual LAI agents. (Full models and results are available from the authors upon request.) Our analyses did not make corrections for multiple comparisons because, given the limited evidence base in this population, we preferred to explore leads that may turn out to be wrong rather than miss potentially important findings, as suggested by Rothman et al. (1990). ${ }^{42}$

\section{Results}

The final sample included 3,768 patients. Of these patients, 91\% ( $\mathrm{n}=3,428)$ received oral antipsychotics, and 9.0\% ( $\mathrm{n}=340)$ received LAI antipsychotics after hospital discharge. Slightly over half ( $\mathrm{n}=183,53.8 \%)$ of the LAI initiators used an SGA LAI. The most frequently used LAI was haloperidol decanoate $(\mathrm{n}=112,32.9 \%)$, closely followed by paliperidone palmitate $(n=102,30 \%)$. About a quarter $(23.8 \%)$ of the patients used risperidone LAI, whereas use of fluphenazine decanoate was limited (13.2\%).

Baseline characteristics of oral versus LAI antipsychotic users, overall and by FGA/SGA status, are displayed in Table 1. Parallel information for users of oral versus individual LAI antipsychotic agents is shown in Table 2. Differences in several sociodemographic characteristics were observed across groups. Of note, the group receiving LAIs had a higher percentage of males when compared with those receiving oral antipsychotics. While no significant differences in mean age were observed, the median age of patients receiving SGA LAIs was lower than that of patients receiving oral antipsychotics. Racial differences were also evident, albeit mixed by type of LAI agent. Patients on FGA LAIs were more likely, whereas patients on SGA LAIs were less likely, to be in capitated Medicaid plans compared with patients on oral antipsychotics.

Patients on LAIs had a slightly lower mean number of comorbidities compared with patients on oral antipsychotics. There were no differences in the prevalence of cardiometabolic comorbidities and substance abuse disorders across the groups. However, patients on LAIs were less likely to have diagnoses of, or use of medications for, other psychiatric disorders, particularly depression, in the 6-month pre-index period. On the other hand, the likelihood of having any 60-day continuous medication gaps or any hospitalization in the 6-month pre- 


\section{Antipsychotic Adherence and Rehospitalization in Schizophrenia Patients Receiving Oral Versus Long-Acting Injectable Antipsychotics Following Hospital Discharge}

TABLE 2 Baseline Characteristics of Patients with Schizophrenia Receiving Oral Antipsychotic Medications Versus Individual LAl Agents

\begin{tabular}{|c|c|c|c|c|c|c|c|c|c|c|c|c|c|c|}
\hline \multirow[b]{2}{*}{ Variable } & \multicolumn{2}{|c|}{$\begin{array}{c}\text { Orals } \\
(\mathrm{n}=3,428)\end{array}$} & \multicolumn{3}{|c|}{$\begin{array}{c}\text { Fluphenazine LAI } \\
(\mathrm{n}=45)\end{array}$} & \multicolumn{3}{|c|}{$\begin{array}{c}\text { Haloperidol LAI } \\
(\mathrm{n}=112)\end{array}$} & \multicolumn{3}{|c|}{$\begin{array}{c}\text { Risperidone LAI } \\
(\mathbf{n}=81)\end{array}$} & \multicolumn{3}{|c|}{$\begin{array}{l}\text { Paliperidone LAI } \\
(\mathrm{n}=102)\end{array}$} \\
\hline & $\%$ & (n) & $\%$ & $(\mathrm{n})$ & $P$ Value & $\%$ & $(\mathrm{n})$ & $P$ Value & $\%$ & (n) & $P$ Value & $\%$ & (n) & $P$ Value \\
\hline \multicolumn{15}{|l|}{ Age, years } \\
\hline Mean [SD] & 38.0 & [12.9] & 41.5 & [12.3] & 0.060 & 37.6 & {$[13.0]$} & 0.751 & 37.3 & [15.3] & 0.703 & 35.8 & {$[13.8]$} & 0.097 \\
\hline Median [IQR] & 37.0 & {$[23.0]$} & 43.0 & {$[18.0]$} & 0.072 & 36.0 & {$[22.0]$} & 0.739 & 32.0 & {$[26.0]$} & 0.406 & 32.0 & {$[22.0]$} & 0.061 \\
\hline Gender & & & & & 0.232 & & & 0.054 & & & 0.101 & & & $<0.001$ \\
\hline Female & 51.2 & $(1,755)$ & 42.2 & (19) & & 42.0 & $(47)$ & & 42.0 & (34) & & 32.4 & (33) & \\
\hline Male & 48.8 & $(1,673)$ & 57.8 & $(26)$ & & 58.0 & $(65)$ & & 58.0 & $(47)$ & & 67.6 & (69) & \\
\hline Race & & & & & 0.273 & & & 0.002 & & & 0.122 & & & 0.053 \\
\hline African American & 48.2 & $(1,652)$ & 62.2 & $(28)$ & & 66.1 & $(74)$ & & 51.9 & $(42)$ & & 42.2 & $(43)$ & \\
\hline White & 42.6 & $(1,460)$ & 31.1 & (14) & & 24.1 & $(27)$ & & 37.0 & $(30)$ & & 46.1 & $(47)$ & \\
\hline Hispanic & 1.1 & (37) & 0.0 & $(0)$ & & 0.9 & (1) & & 3.7 & (3) & & 3.9 & (4) & \\
\hline Other & 8.1 & $(279)$ & 6.7 & (3) & & 8.9 & (10) & & 7.4 & (6) & & 7.8 & $(8)$ & \\
\hline Type of Medicaid plan & & & & & 0.050 & & & 0.131 & & & 0.612 & & & $<0.001$ \\
\hline Fee-for-service & 58.9 & $(2,019)$ & 44.4 & $(20)$ & & 51.8 & $(58)$ & & 61.7 & $(50)$ & & 76.5 & $(78)$ & \\
\hline Capitated & 41.1 & $(1,409)$ & 55.6 & $(25)$ & & 48.2 & $(54)$ & & 38.3 & $(31)$ & & 23.5 & $(24)$ & \\
\hline \multicolumn{15}{|l|}{ Number of Elixhauser comorbidities, pre-index } \\
\hline Mean [SD] & 2.8 & {$[2.2]$} & 2.7 & {$[2.3]$} & 0.637 & 2.4 & [1.9] & 0.020 & 2.8 & {$[2.4]$} & 0.789 & 2.3 & {$[1.8]$} & 0.004 \\
\hline Median [IQR] & 2.0 & [3.0] & 2.0 & {$[3.0]$} & 0.447 & 2.0 & {$[2.0]$} & 0.018 & 2.0 & {$[3.0]$} & 0.478 & 2.0 & {$[2.0]$} & 0.020 \\
\hline \multicolumn{15}{|l|}{ Specific comorbidities, pre-index } \\
\hline Diabetes/hypertension/circulatory disorders & 39.0 & $(1,337)$ & 37.8 & $(17)$ & 0.867 & 37.5 & $(42)$ & 0.748 & 37.0 & $(30)$ & 0.720 & 30.4 & (31) & 0.079 \\
\hline Injury & 31.2 & $(1,069)$ & 24.4 & (11) & 0.332 & 18.8 & (21) & 0.005 & 33.3 & (27) & 0.680 & 27.5 & (28) & 0.422 \\
\hline Bipolar disorder & 34.1 & $(1,170)$ & 22.2 & (10) & 0.094 & 26.8 & (30) & 0.106 & 23.5 & (19) & 0.045 & 30.4 & (31) & 0.432 \\
\hline Depression & 39.2 & $(1,344)$ & 20.0 & (9) & 0.009 & 30.4 & (34) & 0.059 & 27.2 & $(22)$ & 0.028 & 22.5 & $(23)$ & $<0.001$ \\
\hline Anxiety & 34.4 & $(1,178)$ & 15.6 & (7) & 0.008 & 23.2 & (26) & 0.014 & 27.2 & (22) & 0.177 & 25.5 & (26) & 0.062 \\
\hline Substanc & 44.0 & $(1,507)$ & 42.2 & (19) & 0.815 & 46.4 & (52) & 0.605 & 43.2 & (35) & 0.893 & 47.1 & (48) & 0.535 \\
\hline Suicidal behavior and/or ideation & 17.3 & (593) & 0.0 & (0) & 0.003 & 15.2 & $(17)$ & 0.559 & 8.6 & (7) & 0.041 & 11.8 & (12) & 0.144 \\
\hline \multicolumn{15}{|l|}{ Oral antipsychotic adherence, pre-index } \\
\hline Mean PDC [SD] & 0.28 & {$[0.28]$} & 0.26 & {$[0.28]$} & 0.489 & 0.19 & {$[0.24]$} & $<0.001$ & 0.22 & {$[0.26]$} & 0.038 & 0.27 & {$[0.28]$} & 0.694 \\
\hline Any 60-day gap & 69.8 & $(2,394)$ & 73.3 & $(33)$ & 0.803 & 82.1 & $(92)$ & 0.253 & 81.5 & $(66)$ & 0.368 & 71.6 & $(73)$ & 0.598 \\
\hline \multicolumn{15}{|l|}{ Oral antipsychotic agents used, pre-index } \\
\hline Fluphenazine oral & 1.2 & $(40)$ & 6.7 & (3) & 0.013 & 3.6 & (4) & 0.051 & 0.0 & (0) & 0.627 & 1.0 & (1) & 0.999 \\
\hline Haloperidol oral & 7.9 & $(270)$ & 2.2 & (1) & 0.185 & 18.8 & (21) & $<0.001$ & 2.5 & (2) & 0.081 & 10.8 & (11) & 0.285 \\
\hline Other FGA oral & 5.2 & (179) & 4.4 & (2) & 0.999 & 5.4 & (6) & 0.949 & 2.5 & (2) & 0.310 & 8.8 & (9) & 0.110 \\
\hline Olanzapine oral & 8.7 & (297) & 13.3 & (6) & 0.270 & 7.1 & (8) & 0.572 & 7.4 & (6) & 0.691 & 11.8 & (12) & 0.275 \\
\hline Paliperidone oral & 4.2 & (144) & 0.0 & $(0)$ & 0.275 & 1.8 & (2) & 0.224 & 1.2 & (1) & 0.267 & 9.8 & (10) & 0.006 \\
\hline Quetiapine oral & 20.0 & $(684)$ & 24.4 & (11) & 0.454 & 13.4 & (15) & 0.086 & 17.3 & (14) & 0.552 & 12.7 & (13) & 0.072 \\
\hline Ziprasidone oral & 9.0 & (310) & 8.9 & (4) & 0.999 & 6.3 & (7) & 0.308 & 2.5 & (2) & 0.049 & 7.8 & (8) & 0.677 \\
\hline Aripiprazole oral & 9.8 & (336) & 8.9 & (4) & 0.999 & 4.5 & (5) & 0.060 & 6.2 & (5) & 0.276 & 8.8 & (9) & 0.743 \\
\hline Risperidone oral & 20.8 & (713) & 15.6 & (7) & 0.389 & 16.1 & (18) & 0.224 & 34.6 & $(28)$ & 0.003 & 20.6 & $(21)$ & 0.959 \\
\hline Other SGA oral & 3.1 & $(107)$ & 2.2 & $(1)$ & 0.999 & 2.7 & (3) & 0.999 & 3.7 & (3) & 0.999 & 3.9 & $(4)$ & 0.769 \\
\hline \multicolumn{15}{|l|}{ Other CNS drug use, pre-index } \\
\hline Other MH drug use $\mathrm{b}^{\mathrm{b}}$ & 59.3 & $(2,032)$ & 42.2 & (19) & 0.021 & 47.3 & $(53)$ & 0.011 & 51.9 & $(42)$ & 0.179 & 49.0 & $(50)$ & 0.038 \\
\hline Anticholinergic use & 21.3 & $(731)$ & 26.7 & (12) & 0.385 & 32.1 & (36) & 0.006 & 17.3 & (14) & 0.379 & 23.5 & $(24)$ & 0.593 \\
\hline Any MH hospitalization, pre-index & 19.8 & $(680)$ & 11.1 & (5) & 0.144 & 22.3 & $(25)$ & 0.517 & 14.8 & (12) & 0.262 & 13.7 & (14) & 0.126 \\
\hline Any non-MH hospitalization, pre-index & 12.5 & $(429)$ & 11.1 & (5) & 0.777 & 7.1 & (8) & 0.089 & 13.6 & (11) & 0.775 & 8.8 & (9) & 0.265 \\
\hline Year of index hospitalization & & & & & 0.200 & & & 0.778 & & & 0.422 & & & 0.018 \\
\hline 2010 & 39.5 & $(1,353)$ & 51.1 & $(23)$ & & 42.0 & $(47)$ & & 45.7 & $(37)$ & & 26.5 & $(27)$ & \\
\hline 2011 & 30.8 & $(1,055)$ & 20.0 & (9) & & 31.3 & $(35)$ & & 24.7 & $(20)$ & & 33.3 & $(34)$ & \\
\hline 2012 & 29.8 & $(1,020)$ & 28.9 & (13) & & 26.8 & (30) & & 29.6 & (24) & & 40.2 & (41) & \\
\hline \multicolumn{15}{|l|}{ Index hospitalization length of stay, days } \\
\hline Mean [SD] & 8.9 & {$[9.2]$} & 13.7 & {$[8.6]$} & $<0.001$ & 11.5 & {$[8.5]$} & 0.003 & 12.1 & {$[8.8]$} & 0.002 & 14.5 & [17.6] & 0.002 \\
\hline Median [IQR] & 7.0 & [5.0] & 10.0 & [12.0] & $<0.001$ & 9.0 & {$[6.0]$} & $<0.001$ & 8.0 & {$[8.0]$} & $<0.001$ & 11.0 & {$[9.0]$} & $<0.001$ \\
\hline \multicolumn{15}{|c|}{$\begin{array}{l}\text { Includes patients receiving an antipsychotic medication within } 30 \text { days of discharge from index schizophrenia-related hospitalization. Baseline refers to the index hospital- } \\
\text { ization date and the prior } 6 \text {-month pre-index period. P values were calculated with chi-square tests for categorical variables, } t \text {-tests or Fisher's exact tests (for cell sizes less } \\
\text { than 5) for continuous variables, and Wilcoxon tests for medians. } \\
\text { bIncludes antidepressants, anxiolytics, mood stabilizers, sedatives, and hypnotics. } \\
C N S=\text { central nervous system; FGA =first-generation antipsychotic; IQR=interquartile range; LAI=long-acting injectable; MH=mental health; PDC=proportion of days } \\
\text { covered; SD = standard deviation; SGA=second-generation antipsychotic. }\end{array}$} \\
\hline
\end{tabular}


A. $\mathrm{PDC}<0.80$

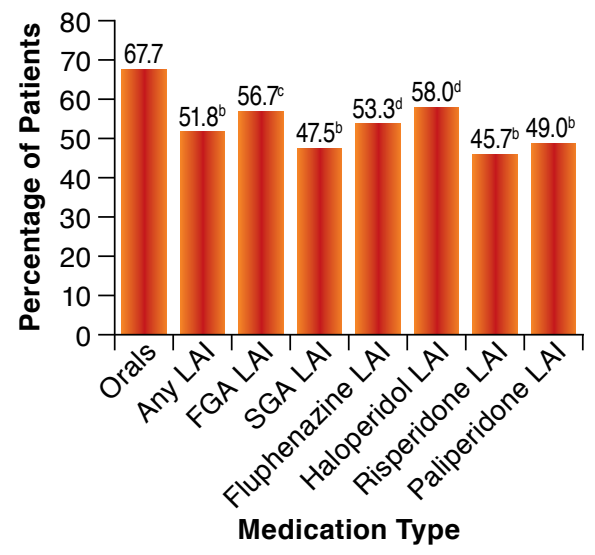

B. 60-Day Gap

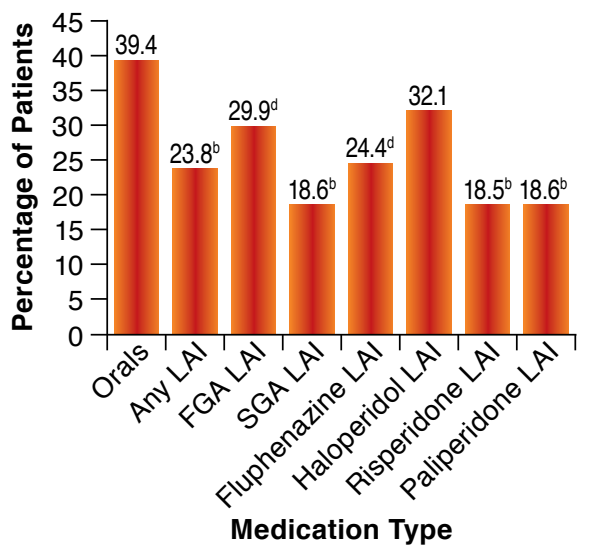

C. Rehospitalization for Schizophrenia

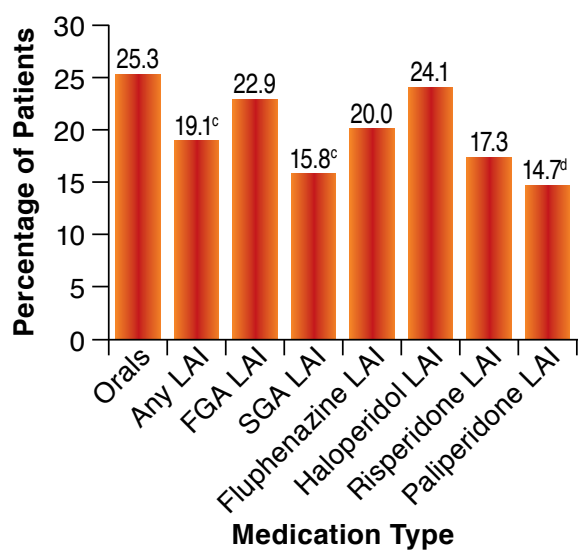

ancludes patients receiving an oral or LAI antipsychotic medication within 30 days of discharge from index schizophrenia-related hospitalization. All comparisons use orals as the reference group. $P$ values were calculated using chi-square tests.

${ }^{b} P<0.001$.

${ }^{c} P \leq 0.01$

$d P<0.05$.

FGA = first-generation antipsychotic; LAI=long-acting injectable; PDC=proportion of days covered; SGA =second-generation antipsychotic.

index period was no different between patients receiving LAIs and those receiving oral antipsychotics, although mean PDC was slightly lower among users of LAIs (particularly haloperidol decanoate and risperidone LAI) in the 6-month pre-index period. Finally, patients receiving LAIs were more likely to have a longer index hospitalization compared with patients receiving oral antipsychotics.

Figure 2 displays descriptive outcomes during the 6 months following hospital discharge. A smaller percentage of LAI users were nonadherent $(51.8 \%$ vs. $67.7 \%, P<0.001)$; had a 60 -day continuous gap in medication $(23.8 \%$ vs. $39.4 \%, P<0.001)$; and were rehospitalized for schizophrenia (19.1\% vs. $25.3 \%$, $P=0.01)$ compared with patients on oral medications. The size of these differences was magnified when comparing SGA LAI users to those receiving oral antipsychotics for nonadherence (47.5\% vs. $67.7 \%, P<0.001)$; for 60 -day continuous gap $(18.6 \%$ vs. $39.4 \%, P<0.001)$; and for schizophrenia-related rehospitalization ( $15.8 \%$ vs. $25.3 \%, P=0.004)$. When examining individual LAIs, patients on paliperidone palmitate and risperidone LAI had the lowest rates of nonadherence, 60-day continuous gap, and schizophrenia-related rehospitalization.

Logistic regressions controlling for all differences in measured covariates confirmed that LAI users had lower odds of being nonadherent (adjusted odds ratio $[\mathrm{AOR}]=0.35,95 \%$ confidence interval $[\mathrm{CI}]=0.27-0.46, P<0.001)$ and having continuous gaps of 60 days $(\mathrm{AOR}=0.45,95 \% \mathrm{CI}=0.34-0.60$, $P<0.001)$ when compared with patients receiving oral medications (Table 3). Both the FGA and SGA LAI users had lower odds of nonadherence compared with patients using oral antipsychotics. Similarly, the FGA LAI users $(\mathrm{AOR}=0.58,95 \%$ $\mathrm{CI}=0.40-0.85, P=0.005)$ and SGA LAI users $(\mathrm{AOR}=0.34,95 \%$ $\mathrm{CI}=0.23-0.51, P<0.001)$ had lower odds of a 60-day continuous gap compared with patients receiving oral antipsychotics. Sensitivity analyses that examined continuous gaps of 30 days and 90 days showed similar results (Table 4).

Compared with those receiving oral antipsychotics, LAI users had lower odds of rehospitalization $(\mathrm{AOR}=0.73,95 \%$ $\mathrm{CI}=0.54-0.99, P=0.041$ ); however, when examined separately, only patients using SGA LAIs (AOR $=0.59,95 \% \mathrm{CI}=0.38-0.90$, $P=0.015)$ and not FGA LAIs (AOR $=0.90,95 \% \mathrm{CI}=0.60-1.34$, $P=0.599)$ had a statistically significant reduction in odds of rehospitalization (Table 3). Among individual LAIs, only users of paliperidone palmitate had statistically different odds of rehospitalization compared with users of oral antipsychotics (AOR $=0.53,95 \% \mathrm{CI}=0.30-0.94, P=0.031$ ). While odds of rehospitalization were 33\% lower among patients receiving risperidone LAI compared with those receiving oral antipsychotics, the estimate did not reach statistical significance (AOR $=0.67,95 \% \mathrm{CI}=0.37-1.22, P=0.194)$. Sensitivity analyses examining mental health-related hospitalization and all-cause hospitalizations showed similar results (Table 4).

Similar results were also found in sensitivity analyses when FGA orals were compared with FGA LAIs (overall and by individual FGA LAI agent), and SGA orals were compared with SGA LAIs (overall and by individual SGA LAI agent; see Appendices A and B, available in online article). 


\section{Antipsychotic Adherence and Rehospitalization in Schizophrenia Patients Receiving Oral Versus Long-Acting Injectable Antipsychotics Following Hospital Discharge}

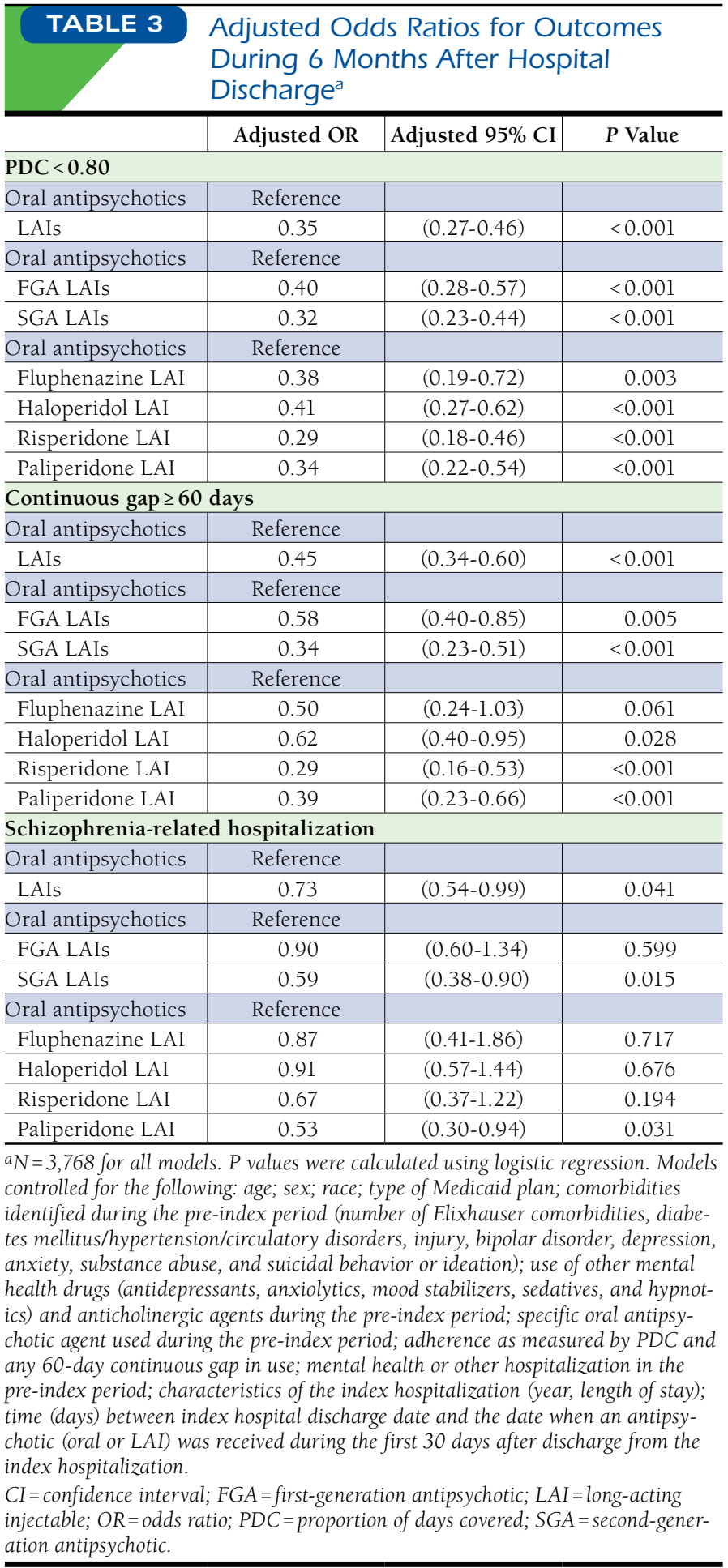

\section{Discussion}

$\overline{\text { This retrospective claims-based study examined posthospital- }}$ ization discharge adherence and rehospitalization outcomes in real-world Medicaid patients receiving either oral or LAI antipsychotic medications. Consistent with clinical guidelines on LAI prescribing, we focused on patients with a history of nonadherence in the 6 months before their index hospitalizations. We examined outcomes for all FGA/SGA LAI medications that were available during the study period. We found that LAI initiation after a schizophrenia-related hospitalization showed advantages over oral medications in all 3 of our outcome measures-nonadherence, medication discontinuation, and rehospitalization-with SGA LAI medications conferring the greatest benefits.

Despite the known clinical and practical benefits of LAIs over oral antipsychotics, they are still prescribed infrequently in clinical practice in the United States. A recent claims-based study found that $15 \%$ of patients (regardless of their nonadherence history) were initiated on an LAI antipsychotic, ${ }^{26}$ and prior studies that focused specifically on patients with recent nonadherence documented that between 19\% and 30\% of patients received LAIs. ${ }^{43-45}$ Prior investigations regarding the reasons for low rates of use have uncovered clinician and patient barriers to LAI prescribing, including physician perceptions that patients are already adherent to oral medication and patient concerns about injections. ${ }^{46-48}$ Several studies have found that psychiatrists discuss LAI treatment with only 30\%-50\% of their patients, yet there is also evidence that many patients are amenable to LAI use once their concerns are addressed. ${ }^{47,48}$ Given that hospitalization provides an opportunity to revisit treatment options, and that our study examined patients who might be most appropriate for LAI initiation (i.e., those being discharged from a hospitalization for schizophrenia, with documented nonadherence in the 6 months prior), it is notable that only $9 \%$ of individuals in our sample received an LAI within 30 days after hospital discharge. Furthermore, only slightly more than half of these patients were initiated on an SGA LAI. Since our data source did not include information about formularies or utilization management policies, it is unclear to what extent such policies may have created additional barriers to LAI use.

To our knowledge, this is the first claims-based study to compare oral and LAI antipsychotics (by FGA/SGA LAI status and by individual LAI agent) in a multistate Medicaid population after the introduction of more recent SGA LAIs such as paliperidone palmitate. Indeed, paliperidone palmitate showed the greatest advantage in terms of being the only agent to be associated with a statistically significant reduction in risk for schizophrenia-related rehospitalizations, although the ability to detect a similar statistically significant advantage for risperidone LAI may have been limited by its smaller sample size. Our sample did not include any eligible patients initiated on olanzapine pamoate, which is currently available only through a restricted distribution program, and the most recently available SGA LAI, aripiprazole LAI, was approved in 2013, after our study sample selection period. ${ }^{36}$ Future studies are needed to examine the benefits conferred by the avail- 


\section{Antipsychotic Adherence and Rehospitalization in Schizophrenia Patients Receiving Oral Versus Long-Acting Injectable Antipsychotics Following Hospital Discharge}

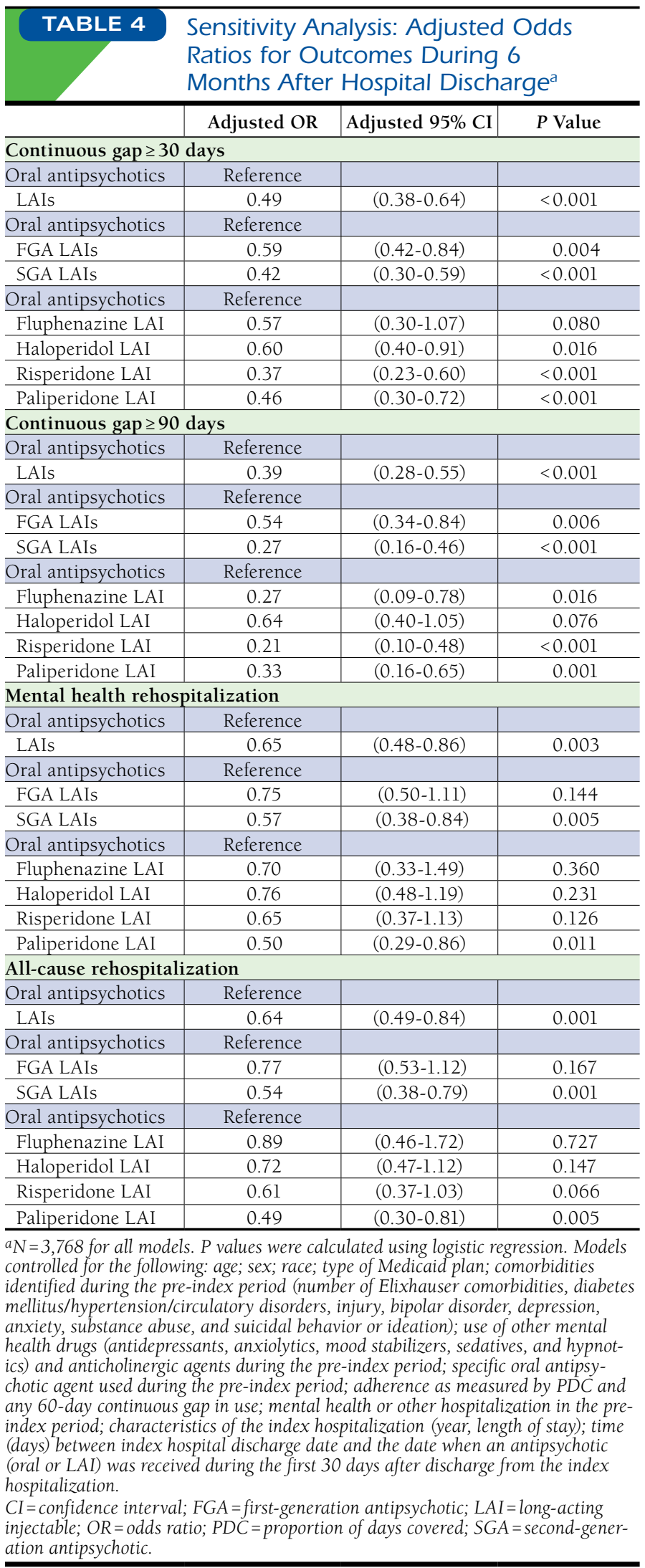

ability of a broad spectrum of LAI formulations of the newer atypical antipsychotics.

The prevention of relapses warranting hospitalization is important for 2 key reasons. First, the associated decline in functioning is disruptive to individuals and their loved ones, and multiple relapses are associated with more negative longterm outcomes. ${ }^{49}$ Second, the increased financial costs associated with frequent rehospitalizations are well documented.,50 Relapse has been linked to higher inpatient costs as well to higher costs for outpatient services and medication. ${ }^{50}$ Thus, our findings on reduced risk of schizophrenia-related rehospitalizations among patients initiating LAIs, particularly SGA LAIs relative to orals, suggest that such LAIs may be associated with lower inpatient costs and potentially other downstream health care cost savings. This has direct implications for payer efforts to control overall health care costs, given that more restrictive formularies or utilization management policies focused on reducing pharmacy costs may inadvertently increase overall health care costs in the long run. Future studies should examine the medical cost savings associated with initiating recently hospitalized nonadherent patients on LAIs relative to oral antipsychotic agents and the extent to which such savings may offset the higher costs of SGA LAIs to inform prescription policies impacting access to LAIs in this vulnerable patient population.

Overall, our results are consistent with findings of other observational, administrative database studies comparing LAIs with oral antipsychotics. ${ }^{26-28,30}$ Further, our study design addressed several factors that can complicate interpretation of prior studies comparing oral and LAI medications. Since practice guidelines recommend LAI therapy specifically for patients with a history of nonadherence who may also have greater illness severity, patients on oral and LAI therapy may have inherent differences that make it more difficult to demonstrate improved outcomes (i.e., lower risk of nonadherence and rehospitalizations) for LAIs in comparison with oral medications. By requiring that all patients in our study have a recent history of nonadherence and a recent schizophreniarelated hospitalization, we sought to obtain greater equivalence between the oral and LAI treatment groups.

\section{Limitations}

Our study has several limitations that deserve mention. While our sample selection approach attempted to minimize underlying differences across patients receiving oral and LAI antipsychotics, several differences remained in the observed characteristics across the groups. We used multivariate regressions, which have been shown to produce similar results to propensity score approaches in controlling for such observed confounding, to address this. ${ }^{51,52}$ Furthermore, we conducted a series of sensitivity analyses that confirmed the robustness of our findings. Nevertheless, our study shares the limitation of unobserved confounding present in observational studies, 


\section{Antipsychotic Adherence and Rehospitalization in Schizophrenia Patients Receiving Oral Versus Long-Acting Injectable Antipsychotics Following Hospital Discharge}

especially given that retrospective claims data do not include additional clinical severity measures (e.g., symptom rating scales) or self-reported patient measures (e.g., adherence and attitudes toward medication) that may have allowed for further detection and control of potential differences in illness severity or adherence behavior between patient groups. Similarly, given the limited length of the pre-index period in claimsbased studies, data on duration of illness or prior treatment response were not available. Furthermore, for de-identification purposes, the dataset did not contain state identifiers or any other geographic information. As a result, we were unable to assess or control for regional or other differences, such as utilization review policies or formulary restrictions that may have impacted access to specific medications within individual state Medicaid programs.

As previously noted, it is possible that access issues contributed to the relatively small sample of LAI initiators among eligible postdischarge patients and limited our statistical power to detect differences for specific LAIs, as well as to conduct head-to-head comparisons between FGA and SGA LAIs. In some cases, restrictions in access could also mean that only treatment-resistant patients or those with more severe impairment would have access to LAIs. To the extent that these unobserved confounders resulted in more severe patients being initiated on LAIs, our findings may be an underestimate of true differences between groups. The reverse could also be true if patients more likely to have poorer outcomes were initiated on oral antipsychotics.

Adherence measures calculated from prescription claims data may also underestimate the comparative clinical benefits of LAIs for medication adherence. Such measures offer a "best case scenario" for oral medication use by evaluating whether patients had sufficient medication supply to cover each day in the observation period; however, it is unknown if patients actually consumed the medication. Similarly, since not all clinically significant exacerbations in symptoms or relapses will result in hospitalization, our use of hospitalization as a marker of relapse offers a limited view of potential differences in the outcomes associated with use of LAIs versus oral medications. Nevertheless, from a payer perspective, hospitalizations are the most costly and policy-relevant outcomes. ${ }^{50}$ In addition, as is the case with all claims-based studies, data were collected for administrative purposes and may be subject to coding errors. And, while our focus on Medicaid patients is a strength of our study, given the proportion of schizophrenia patients covered under Medicaid, our findings may be limited in their generalizability to other insured populations.

\section{Conclusions}

By including more recently approved SGA LAIs, focusing on the Medicaid population, and restricting our study population to patients with a recent history of nonadherence, this claims-based analysis of posthospital discharge adherence and rehospitalization outcomes contributes to the growing real-world evidence base of the benefits of LAI antipsychotic medications in routine practice, especially with regard to SGA LAIs. Patients initiated on LAI medications, particularly SGA LAIs, showed lower odds of all 3 key outcomes: nonadherence (as measured by PDC), discontinuation (defined as a 60-day continuous gap in available medication), and schizophreniarelated rehospitalization in the 6 months following an index hospitalization for schizophrenia, compared with patients receiving oral medications. Our findings provide important information for payers and clinicians treating patients with schizophrenia in real-world clinical practice. As new SGA LAI formulations become available, future real-world studies with larger sample sizes will be needed to further delineate their potential advantages in regard to both outcomes and costs.

\section{Authors}

STEVEN C. MARCUS, PhD, is Research Associate Professor, School of Social Policy \& Practice, University of Pennsylvania, Philadelpha. JACQUELINE ZUMMO, MPH, MBA, is Director, Medical and Scientific Communications, and JEFFREY STODDARD, MD, is Vice President, Medical Professional Services, Alkermes, Inc., Waltham, Massachusetts. AMY R. PETTIT, PhD, is an Independent Consultant, Boston, Massachusetts, and JALPA A. DOSHI, PhD, is Associate Professor, Perelman School of Medicine, University of Pennsylvania, Philadelphia.

AUTHOR CORRESPONDENCE: Steven C. Marcus, PhD, Research Associate Professor, School of Social Policy \& Practice, University of Pennsylvania, 3701 Locust Walk, Philadelphia, PA 19104-6214. Tel:. 215.573.7941; E-mail:marcuss@upenn.edu.

\section{DISCLOSURES}

This study was funded by Alkermes, Inc. Marcus, Pettit, and Doshi report serving as consultants to Alkermes. Stoddard and Zummo are employees of Alkermes.

Study concept and design were contributed by Marcus, Doshi, Zummo, and Stoddard. Data collection was carried out by Zummo, Stoddard, and Marcus, and data interpretation was performed by Marcus, Doshi, Pettit, Zummo, and Stoddard. The manuscript was written by Marcus, Doshi, and Pettit, with assistance from Zummo and Stoddard, and revised by Zummo, Stoddard, and Pettit, along with Marcus and Doshi.

\section{REFERENCES}

1. Simpson GM. Atypical antipsychotics and the burden of disease. Am J Manag Care. 2005;11(8 Suppl):S235-41. Available at: http://www.ajmc com/publications/supplement/2005/2005-09-voll1-n8Suppl/Sep052135pS235-S241/. Accessed July 16, 2015

2. Rice DP. The economic impact of schizophrenia. J Clin Psychiatry. 1999;60(Suppl 1):4-6

3. Wu EQ, Birnbaum HG, Shi L, et al. The economic burden of schizophrenia in the United States in 2002. J Clin Psychiatry. 2005;66(9):1122-29.

4. Olfson M, Mechanic D, Hansell S, Boyer CA, Walkup J, Weiden PJ. Predicting medication noncompliance after hospital discharge among patients with schizophrenia. Psychiatr Serv. 2000;51(2):216-22. 


\section{Antipsychotic Adherence and Rehospitalization in Schizophrenia Patients Receiving Oral Versus Long-Acting Injectable Antipsychotics Following Hospital Discharge}

5. Lehman AF, Lieberman JA, Dixon LB, et al. Practice guideline for the treatment of patients with schizophrenia, second edition. Am J Psychiatry. 2004;161(2 Suppl):1-56

6. Lehman AF, Kreyenbuhl J, Buchanan RW, et al. The Schizophrenia Patient Outcomes Research Team (PORT): updated treatment recommendations 2003. Schizophr Bull. 2004;30(2):193-217. Available at: http://schizophreniabulletin.oxfordjournals.org/content/30/2/193.long. Accessed July 16, 2015.

7. Ascher-Svanum H, Zhu B, Faries DE, Lacro JP, Dolder CR, Peng X. Adherence and persistence to typical and atypical antipsychotics in the naturalistic treatment of patients with schizophrenia. Patient Prefer Adherence. 2008;2:67-77. Available at: http://www.ncbi.nlm.nih.gov/pmc/articles/ PMC2770396/pdf/ppa-2-67.pdf. Accessed July 16, 2015.

8. Cramer JA, Rosenheck R. Enhancing medication compliance for people with serious mental illness. J Nerv Ment Dis. 1999;187(1):53-55.

9. Lieberman JA, Stroup TS, McEvoy JP, et al. Effectiveness of antipsychotic drugs in patients with chronic schizophrenia. N Engl J Med. 2005;353(12):1209-23. Available at: http://www.nejm.org/doi/full/10.1056/ NEJMoa051688. Accessed July 16, 2015.

10. Gilmer TP, Dolder CR, Lacro JP, et al. Adherence to treatment with antipsychotic medication and health care costs among Medicaid beneficiaries with schizophrenia. Am J Psychiatry. 2004;161(4):692-99.

11. Haywood TW, Kravitz HM, Grossman LS, Cavanaugh JL Jr, Davis JM, Lewis DA. Predicting the "revolving door" phenomenon among patients with schizophrenic, schizoaffective, and affective disorders. Am J Psychiatry. 1995;152(6):856-61

12. Weiden PJ, Kozma C, Grogg A, Locklear J. Partial compliance and risk of rehospitalization among California Medicaid patients with schizophrenia. Psychiatr Serv. 2004;55(8):886-91

13. Simpson GM. A brief history of depot neuroleptics. J Clin Psychiatry. 1984:45(5 Pt 2):3-4.

14. Adams CE, Fenton MK, Quraishi S, David AS. Systematic meta-review of depot antipsychotic drugs for people with schizophrenia. Br J Psychiatry. 2001;179(4):290-99. Available at: http://bjp.rcpsych.org/content/179/4/290 long. Accessed July 16, 2015.

15. Donovan JL, Blake DR. Patient non-compliance: deviance or reasoned decision-making? Soc Sci Med. 1992;34(5):507-13.

16. Raskin SA, Maye J, Rogers A, Correll D, Zamroziewicz M, Kurtz M. Prospective memory in schizophrenia: relationship to medication management skills, neurocognition, and symptoms in individuals with schizophrenia. Neuropsychology. 2014;28(3):359-65.

17. Velligan DI, Wang M, Diamond P, et al. Relationships among subjective and objective measures of adherence to oral antipsychotic medications. Psychiatr Serv. 2007;58(9):1187-92.

18. Velligan DI, Weiden PJ, Sajatovic M, et al. Strategies for addressing adherence problems in patients with serious and persistent mental illness: recommendations from the expert consensus guidelines. J Psychiatr Pract. 2010;16(5):306-24

19. Kishimoto T, Robenzadeh A, Leucht C, et al. Long-acting injectable vs oral antipsychotics for relapse prevention in schizophrenia: a meta-analysis of randomized trials. Schizophr Bull. 2014;40(1):192-213.

20. Kane JM, Kishimoto T, Correll CU. Assessing the comparative effectiveness of long-acting injectable vs. oral antipsychotic medications in the prevention of relapse provides a case study in comparative effectiveness research in psychiatry. J Clin Epidemiol. 2013;66(8 Suppl):S37-41. Available at: http://www.jclinepi.com/article/S0895-4356\%2813\%2900161-3/fulltext. Accessed July 16, 2015.

21. Zarin DA, Young JL, West JC. Challenges to evidence-based medicine: a comparison of patients and treatments in randomized controlled trials with patients and treatments in a practice research network. Soc Psychiatry Psychiatr Epidemiol. 2005;40(1):27-35.
22. Kane JM. Strategies for improving compliance in treatment of schizophrenia by using a long-acting formulation of an antipsychotic: clinical studies. J Clin Psychiatry. 2003;64(Suppl 16):34-40.

23. Buckley PF, Schooler NR, Goff DC, et al. Comparison of SGA oral medications and a long-acting injectable SGA: the PROACTIVE study. Schizophr Bull. 2015;41(2):449-59.

24. RISPERDAL CONSTA (risperidone) long-acting injection. Janssen Pharmaceuticals, Inc. Revised April 2014. Available at: https://www.risperdalconsta.com/sites/default/files/RisperdalConstaPI.pdf. Accessed July 16, 2015.

25. Kishimoto T, Nitta M, Borenstein M, Kane JM, Correll CU. Long-acting injectable versus oral antipsychotics in schizophrenia: a systematic review and meta-analysis of mirror-image studies. J Clin Psychiatry. 2013;74(10):957-65.

26. Offord S, Wong B, Mirski D, Baker RA, Lin J. Healthcare resource usage of schizophrenia patients initiating long-acting injectable antipsychotics vs. oral. J Med Econ. 2013;16(2):231-39.

27. Lafeuille MH, Laliberté-Auger F, Lefebvre P, Frois C, Fastenau J, Duh MS. Impact of atypical long-acting injectable versus oral antipsychotics on rehospitalization rates and emergency room visits among relapsed schizophrenia patients: a retrospective database analysis. BMC Psychiatry. 2013;13:221. Available at: http://www.ncbi.nlm.nih.gov/pmc/articles/ PMC3847215/. Accessed July 16, 2015.

28. Tiihonen J, Haukka J, Taylor M, Haddad PM, Patel MX, Korhonen P. A nationwide cohort study of oral and depot antipsychotics after first hospitalization for schizophrenia. Am J Psychiatry. 2011;168(6):603-09.

29. Lang K, Meyers JL, Korn JR, Lee S, Sikirica M, Crivera C, Dirani R, Menzin J. Medication adherence and hospitalization among patients with schizophrenia treated with antipsychotics. Psychiatr Serv. 2010;61(12):1239-47.

30. Morrato EH, Parks J, Campagna EJ, et al. Comparative effectiveness of injectable paliperidone palmitate versus oral atypical antipsychotics: early postmarketing evidence. J Comp Eff Res. 2015;4(2):89-99.

31. INVEGA SUSTENNA (paliperidone palmitate) extended-release injectable suspension, for intramuscular use. Janssen Pharmaceuticals, Inc. Revised June 2015. Available at: http://www.janssencns.com/shared/product/invegasustenna/prescribing-information.pdf. Accessed July 16, 2015.

32. Root ED, Thomas DS, Campagna EJ, Morrato EH. Adjusting for geographic variation in observational comparative effectiveness studies: a case study of antipsychotics using state Medicaid data. BMC Health Serv Res. 2014;14:355. Available at: http://www.ncbi.nlm.nih.gov/pmc/articles/ PMC4161848/. Accessed July 16, 2015.

33. Wu EQ, Shi L, Birnbaum H, Hudson T, Kessler R. Annual prevalence of diagnosed schizophrenia in the USA: a claims data analysis approach. Psychol Med. 2006;36(11):1535-40.

34. Lurie N, Popkin M, Dysken M, Moscovice I, Finch M. Accuracy of diagnoses of schizophrenia in Medicaid claims. Hosp Community Psychiatry. 1992:43(1):69-71.

35. ZYPREXA RELPREVV (olanzapine) for extended release injectable suspension. Eli Lilly and Company. Revised December 2014. Available at: http://pi.lilly.com/us/zyprexa_relprevv.pdf. Accessed July 16, 2015.

36. ABILIFY MAINTENA (aripiprazole) for extended-release injectable suspension, for intramuscular use. Otsuka America Pharmaceutical, Inc. April 2014. Available at: http://www.fda.gov/downloads/Drugs/DrugSafety/ UCM342207.pdf. Accessed July 16, 2015.

37. Peterson AM, Nau DP, Cramer JA, Benner J, Gwadry-Sridhar F, Nichol M. A checklist for medication compliance and persistence studies using retrospective databases. Value Health. 2007;10(1):3-12

38. Campagna EJ, Muser E, Parks J, Morrato EH. Methodological considerations in estimating adherence and persistence for a long-acting injectable medication. J Manag Care Spec Pharm. 2014;20(7):756-66. Available at: http://www.amcp.org/JMCP/2014/July/18277/1033.html. 


\section{Antipsychotic Adherence and Rehospitalization in Schizophrenia Patients Receiving Oral Versus Long-Acting Injectable Antipsychotics Following Hospital Discharge}

39. Ray WA, Daugherty JR, Meador KG. Effect of a mental health "carveout" program on the continuity of antipsychotic therapy. N Engl J Med. 2003;348(19):1885-94. Available at: http://www.nejm.org/doi/full/10.1056/ NEJMsa020584. Accessed July 16, 2015.

40. Menzin J, Boulanger L, Friedman M, Mackell J, Lloyd JR. Treatment adherence associated with conventional and atypical antipsychotics in a large state Medicaid program. Psychiatr Serv. 2003;54(5):719-23.

41. Elixhauser A, Steiner C, Harris DR, Coffey R. Comorbidity measures for use with administrative data. Med Care. 1998;36(1):8-27.

42. Rothman KJ. No adjustments are needed for multiple comparisons. Epidemiology. 1990;1(1):43-46

43. West JC, Marcus SC, Wilk J, Countis LM, Regier DA, Olfson M. Use of depot antipsychotic medications for medication nonadherence in schizophrenia. Schizophr Bull. 2008;34(5):995-1001.

44. Valenstein M, Copeland LA, Owen R, Blow FC, Visnic S. Adherence assessments and the use of depot antipsychotics in patients with schizophrenia. J Clin Psychiatry. 2001;62(7):545-51.

45. West JC, Wilk JE, Olfson M, et al. Patterns and quality of treatment for patients with schizophrenia in routine psychiatric practice. Psychiatr Serv. 2005;56(3):283-91

46. Kane JM. Attitudinal barriers to prescribing LAI antipsychotics in the outpatient setting: communicating with patients, families, and caregivers. J Clin Psychiatry. 2014;75(12):e33.
47. Heres S, Hamann J, Kissling W, Leucht S. Attitudes of psychiatrists toward antipsychotic depot medication. J Clin Psychiatry. 2006;67(12):1948-53.

48. Potkin S, Bera R, Zubek D, Lau G. Patient and prescriber perspectives on long-acting injectable (LAI) antipsychotics and analysis of in-office discussion regarding LAI treatment for schizophrenia. BMC Psychiatry. 2013;13:261. Available at: http://www.ncbi.nlm.nih.gov/pmc/articles/ PMC3819472/. Accessed July 16, 2015.

49. Remington G, Foussias G, Agid O, Fervaha G, Takeuchi H, Hahn M. The neurobiology of relapse in schizophrenia. Schizophr Res. 2014;152(2-3): 381-90.

50. Ascher-Svanum H, Zhu B, Faries DE, et al. The cost of relapse and the predictors of relapse in the treatment of schizophrenia. BMC Psychiatry. 2010;10:2. Available at: http://www.ncbi.nlm.nih.gov/pmc/articles/ PMC2817695/. Accessed July 16, 2015.

51. Shah BR, Laupacis A, Hux JE, Austin PC. Propensity score methods gave similar results to traditional regression modeling in observational studies: a systematic review. J Clin Epidemiol. 2005;58(6):550-59.

52. Stürmer T, Joshi M, Glynn RJ, Avorn J, Rothman KJ, Schneeweiss S. A review of the application of propensity score methods yielded increasing use, advantages in specific settings, but not substantially different estimates compared with conventional multivariable methods. J Clin Epidemiol. 2006;59(5):437-47. Available at: http://www.ncbi.nlm.nih.gov/pmc/articles/ PMC1448214/. Accessed July 16, 2015. 


\section{Antipsychotic Adherence and Rehospitalization in Schizophrenia Patients Receiving Oral Versus Long-Acting Injectable Antipsychotics Following Hospital Discharge}

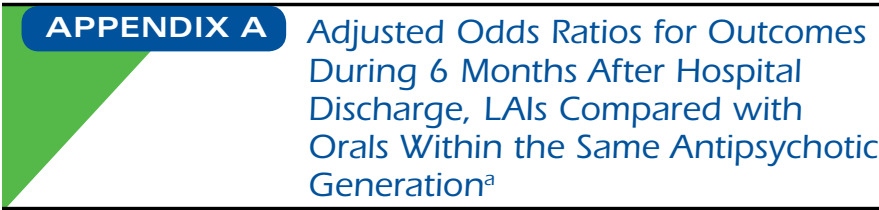

\begin{tabular}{|c|c|c|c|}
\hline & Adjusted OR & Adjusted 95\% CI & $P$ Value \\
\hline \multicolumn{4}{|l|}{ PDC $<0.80$} \\
\hline FGA orals & Reference & & \\
\hline FGA LAIs & 0.34 & $(0.21-0.56)$ & $<0.001$ \\
\hline SGA orals & Reference & & \\
\hline SGA LAIs & 0.32 & $(0.22-0.45)$ & $<0.001$ \\
\hline FGA orals & Reference & & \\
\hline Fluphenazine LAI & 0.31 & $(0.14-0.65)$ & 0.002 \\
\hline Haloperidol LAI & 0.36 & $(0.21-0.61)$ & $<0.001$ \\
\hline SGA orals & Reference & & \\
\hline Risperidone LAI & 0.29 & $(0.18-0.47)$ & $<0.001$ \\
\hline Paliperidone LAI & 0.34 & $(0.22-0.54)$ & $<0.001$ \\
\hline
\end{tabular}

\begin{tabular}{|c|c|c|c|}
\hline \multicolumn{4}{|c|}{ Continuous gap $\geq 60$ days } \\
\hline FGA orals & Reference & & \\
\hline FGA LAIs & 0.43 & $(0.27-0.70)$ & $<0.001$ \\
\hline SGA orals & Reference & & \\
\hline SGA LAIs & 0.36 & $(0.24-0.54)$ & $<0.001$ \\
\hline FGA orals & Reference & & \\
\hline Fluphenazine LAI & 0.37 & $(0.16-0.84)$ & 0.018 \\
\hline Haloperidol LAI & 0.45 & $(0.27-0.77)$ & 0.003 \\
\hline SGA orals & Reference & & \\
\hline Risperidone LAI & 0.31 & $(0.17-0.55)$ & $<0.001$ \\
\hline Paliperidone LAI & 0.41 & $(0.24-0.71)$ & 0.001 \\
\hline
\end{tabular}

\begin{tabular}{l|c|c|c}
\hline \multicolumn{3}{l}{ Schizophrenia-related hospitalization } \\
\hline FGA orals & Reference & & \\
\hline FGA LAIs & 1.01 & $(0.60-1.68)$ & 0.977 \\
\hline SGA orals & Reference & & \\
\hline SGA LAIs & 0.57 & $(0.37-0.88)$ & 0.011 \\
\hline FGA orals & Reference & & \\
\hline Fluphenazine LAI & 0.91 & $(0.38-2.18)$ & 0.831 \\
\hline Haloperidol LAI & 1.04 & $(0.60-1.82)$ & 0.887 \\
\hline SGA orals & Reference & & \\
\hline Risperidone LAI & 0.65 & $(0.36-1.19)$ & 0.163 \\
\hline Paliperidone LAI & 0.51 & $(0.28-0.92)$ & 0.025 \\
\hline
\end{tabular}

aP values were calculated using logistic regression. Each LAI group was compared with orals within its own generation, using a separate model. Models controlled for the following: age; sex; race; type of Medicaid plan; comorbidities identified during the pre-index period number of Elixhauser comorbidities, diabetes mellitus/ hypertension/circulatory disorders, injury, bipolar disorder, depression, anxiety, substance abuse, and suicidal behavior, or ideation); use of other mental health drugs (antidepressants, anxiolytics, mood stabilizers, sedatives, or hypnotics) and anticholinergic agents during the pre-index period; specific oral antipsychotic agent used during the pre-index period; adherence as measured by PDC and any 60-day continuous gap in use; mental health or other hospitalization in the pre-index

period; characteristics of the index hospitalization (year, length of stay); time (days) between index hospital discharge date and the date when an antipsychotic (oral or LAI) was received during the first 30 days after discharge from the index hospitalization.

$C I=$ confidence interval $; F G A=$ first-generation antipsychotic; $L A I=$ long-acting injectable; $O R=$ odds ratio; $P D C=$ proportion of days covered; $S G A=$ second-generation antipsychotic. 


\section{Antipsychotic Adherence and Rehospitalization in Schizophrenia Patients Receiving Oral Versus Long-Acting Injectable Antipsychotics Following Hospital Discharge}

\begin{tabular}{|c|c|c|c|}
\hline \multirow[t]{2}{*}{ APPENDIX } & \multicolumn{3}{|c|}{$\begin{array}{l}\text { Sensitivity Analysis: Adjusted Odds } \\
\text { Ratios for Outcomes During } 6 \\
\text { Months After Hospital Discharge, } \\
\text { LAls Compared with Orals Within } \\
\text { the Same Antipsychotic Generation }{ }^{a}\end{array}$} \\
\hline & Adjusted OR & Adjusted 95\% CI & $P$ Value \\
\hline \multicolumn{4}{|c|}{ Continuous gap $\geq 30$ days } \\
\hline FGA orals & Reference & & \\
\hline FGA LAIs & 0.42 & $(0.27-0.66)$ & $<0.001$ \\
\hline SGA orals & Reference & & \\
\hline SGA LAIs & 0.44 & $(0.31-0.62)$ & $<0.001$ \\
\hline FGA orals & Reference & & \\
\hline Fluphenazine LAI & 0.37 & $(0.18-0.76)$ & 0.007 \\
\hline Haloperidol LAI & 0.34 & $(0.27-0.72)$ & 0.001 \\
\hline SGA orals & Reference & & \\
\hline Risperidone LAI & 0.39 & $(0.24-0.63)$ & $<0.001$ \\
\hline Paliperidone LAI & 0.49 & $(0.31-0.76)$ & 0.002 \\
\hline \multicolumn{4}{|c|}{ Continuous gap $\geq 90$ days } \\
\hline FGA orals & Reference & & \\
\hline FGA LAIs & 0.44 & $(0.25-0.76)$ & 0.003 \\
\hline SGA orals & Reference & & \\
\hline SGA LAIs & 0.28 & $(0.17-0.48)$ & $<0.001$ \\
\hline FGA orals & Reference & & \\
\hline Fluphenazine LAI & 0.22 & $(0.07-0.69)$ & 0.009 \\
\hline Haloperidol LAI & 0.52 & $(0.29-0.93)$ & 0.026 \\
\hline SGA orals & Reference & & \\
\hline Risperidone LAI & 0.22 & $(0.10-0.50)$ & $<0.001$ \\
\hline Paliperidone LAI & 0.34 & $(0.17-0.69)$ & 0.003 \\
\hline \multicolumn{4}{|c|}{ Mental health rehospitalization } \\
\hline FGA orals & Reference & & \\
\hline FGA LAIs & 0.79 & $(0.48-1.29)$ & 0.346 \\
\hline SGA orals & Reference & & \\
\hline SGA LAIs & 0.56 & $(0.37-0.83)$ & 0.004 \\
\hline FGA orals & Reference & & \\
\hline Fluphenazine LAI & 0.69 & $(0.29-1.63)$ & 0.400 \\
\hline Haloperidol LAI & 0.82 & $(0.48-1.41)$ & 0.477 \\
\hline SGA orals & Reference & & \\
\hline Risperidone LAI & 0.64 & $(0.37-1.11)$ & 0.109 \\
\hline Paliperidone LAI & 0.50 & $(0.29-0.85)$ & 0.011 \\
\hline \multicolumn{4}{|c|}{ All-cause rehospitalization } \\
\hline FGA orals & Reference & & \\
\hline FGA LAIs & 0.80 & $(0.50-1.29)$ & 0.362 \\
\hline SGA orals & Reference & & \\
\hline SGA LAIs & 0.54 & $(0.37-0.78)$ & 0.001 \\
\hline FGA orals & Reference & & \\
\hline Fluphenazine LAI & 0.85 & $(0.40-1.83)$ & 0.680 \\
\hline Haloperidol LAI & 0.79 & $(0.47-1.32)$ & 0.366 \\
\hline SGA orals & Reference & & \\
\hline Risperidone LAI & 0.60 & $(0.36-1.02)$ & 0.058 \\
\hline Paliperidone LAI & 0.48 & $(0.29-0.80)$ & 0.005 \\
\hline \multicolumn{4}{|c|}{$\begin{array}{l}\text { aP values were calculated using logistic regression. Each LAI group was compared } \\
\text { with orals within its own class, using a separate model. Models controlled for the } \\
\text { following: age; sex; race; type of Medicaid plan; comorbidities identified during the } \\
\text { pre-index period (number of Elixhauser comorbidities, diabetes mellitus/hyperten- } \\
\text { sion/circulatory disorders, injury, bipolar disorder, depression, anxiety, substance } \\
\text { abuse, and suicidal behavior or ideation); use of other mental health drugs (antide- } \\
\text { pressants, anxiolytics, mood stabilizers, sedatives, or hypnotics) and anticholinergic } \\
\text { agents during the pre-index period; specific oral antipsychotic agent used during } \\
\text { the pre-index period; adherence as measured by PDC and any 60-day continuous } \\
\text { gap in use; mental health or other hospitalization in the pre-index period; char- } \\
\text { acteristics of the index hospitalization (year, length of stay); time (days) between } \\
\text { index hospital discharge date and the date when an antipsychotic (oral or LAI) was } \\
\text { received during the first } 30 \text { days after discharge from the index hospitalization. } \\
C I=\text { confidence interval; FGA = first-generation antipsychotic; } L A I=\text { long-acting } \\
\text { injectable; OR =odds ratio; PDC = proportion of days covered; SGA = second-gener- } \\
\text { ation antipsychotic. }\end{array}$} \\
\hline
\end{tabular}

